This is the accepted manuscript of the article, which has been published in Nanoscale. 2018, 10(41), 19297-19309. http://dx.doi.org/10.1039/C8NR05535A

\title{
DNA-nanoparticle actuator enabling optical monitoring of nanoscale movements induced by electric field
}

Received 00th January 20xx, Accepted 00th January 20xx

DOI: $10.1039 / \times 0 \times x 00000 x$

www.rsc.org/

\author{
Kosti Tapio ${ }^{a}$, Dongkai Shao a†, Sanna Auer ${ }^{\text {bł }}$, Jussi-Pekka Tuppurainenc, M arkus Ahlskog ${ }^{a}$, Vesa P. \\ Hytönen $^{\text {b }}$, J. Jussi Topparia*
}

\begin{abstract}
Merging biological and non-biological matter to fabricate nanoscale assemblies with controllable motion and function are of high interest due to potential application for example in diagnostics and biosensing. Here, we have constructed a DNAbased bionanoactuator that interfaces with biological and non-biological matter via electric field in a reversibly controllable fashion. The read-out of the actuator is based on motion-induced changes in the plasmon resonance of a gold nanoparticle immobilized to a gold surface by single stranded DNA. The motion of the gold nanoparticle and thus the conformational changes of the DNA under varying electric field were analyzed by dark field spectroscopy. After this basic characterization, another actuator was built utilizing hairpin-DNA coated gold nanoparticles, where the hairpin-DNA enabled transition between two specific open-loop and folded-loop states. These two states and the transition dynamics between them were clearly visible in the actuator behavior. The nanoactuator concept could be readily extended to inspection of conformational changes of other biomolecules as well. Besides that, this concept enables other possibilities in applications like surfaceenhanced Raman spectroscopy and fluorescence enhancement, since the specific wavelength of the plasmon resonance of the actuator can be tuned by external voltage.
\end{abstract}

\section{Introduction}

Nature offers excellent building blocks for almost any of the functions and devices used in modern semiconductor-based technology and material science. However, reliable interfaces between the biological and non-biological domains remain to be a challenge. Therefore, bionanoactuators and -sensors have been under intense study during the past decades. Especially, bionanosensors have attracted vast interest in, e.g., environment ${ }^{1,2}$, food 1,3 and biomedical industry 2,4 , where the sensors commonly use either substrate-immobilized or suspended probes, which through either optical ${ }^{5}$, mechanical ${ }^{6}$, electrical7 ${ }^{7}$ or chemical8-10 interaction assay the surrounding medium to extract information. Some more sophisticated nanosensors may require outside activation or actuation via, e.g., solution flow ${ }^{11}$, electric or magnetic fields ${ }^{12}$ or thermooptical effects ${ }^{13}$. If the purpose of the nanodevice is to controllably change the active state of any chemical or physical quantity, rather than to detect any target, it can be called a nanoactuator, even though some kind of sensing system is needed for reading its state.

One of the most interesting molecules for biosensor and actuator applications is DNA due to its robust self-assembly properties, reasonable price and wide selection of available chemical modifications. DNA has been utilized in applications ranging from, e.g., electronics ${ }^{14,15}$ to biosensing16,17, and the immobilization of DNA strands on surfaces has been studied extensively18,19. However, their use in electrical or optical

\footnotetext{
a. University of Jyvaskyla, Department of Physics, Nanoscience Center, P.O. Box 35, FI-40014 University of Jyväskylä, Finland.

b. Faculty of M edicine and Life Sciences and BioM ediTech, University of Tampere,

Arvo Ylpön katu 34, Fl-33520 Tampere, Finland and Fimlab laboratories, Biokatu 4, Fl-33520 Tampere.

c. BioNavis Ltd, Hermiankatu 6-8 H, Fl-33720 Tampere, Finland.

$\dagger$ These authors contributed equally.

‡Present address: BioM ensio Ltd, Hermiankatu 6-8H, Fl-33720 Tampere.

Electronic Supplementary Information (ESI) available: Additional data and analysis, and experimental details. See DOI: 10.1039/x0xx00000x
}

probing is still challenging ${ }^{15,20}$, due to the lack of clear electrical or optical properties. Meanwhile, metallic nanoparticles (NP) have become increasingly popular due to their strong plasmonic and electric properties ${ }^{21}$, enabling even probing of individual particles or structures to obtain information about their state or surroundings 22,23 . In this work, we combine the advantages of the selective binding scheme of biomolecules, especially DNA, to the extremely sensitive optical detection of metallic NPs, so that the system can be reversibly manipulated using external triggers and simultaneously monitored optically.

Here, we present a new type of bionanoactuator based on DNAfunctionalized gold nanoparticles (AuNP), where the plasmonic coupling between the AuNP and a gold surface is used for readout. The charged AuNPs are anchored to the gold surface via DNA linker and moved reversibly by applying electric field to the liquid medium surrounding them. Due to the distancedependent plasmonic coupling between the AuNP and the Ausurface, the localized surface plasmon resonances (LSPR) of the AuNP will either redshift or blueshift. This spectral change is used to determine the distance between the AuNP and the surface, and is further correlated to the conformation of the DNA linker to study its mechanical properties and behavior. We utilized plain single stranded DNA (ssDNA) with varying lengths to calibrate the system, and afterwards a hairpin-DNA to study the folding and unfolding of it under a mechanical load applied via AuNPs dragged by the electric field. This yields information on the unfolding energetics of individual DNA strands.

The demonstrated scheme can be readily extended to study also conformation of other biomolecules by replacing the DNA between the AuNP and surface by any biomolecule, such as talin protein containing relatively soft alpha-helical structures, which greatly elongate under mechanical load in living cells24,25. Also other reasons for conformational changes, like $\mathrm{pH}$ in the case of Influenza Hemagglutinin protein ${ }^{26}$, or could be studied in a similar way. In addition, the plasmonic coupling between AuNP and the surface will induce high electric fields, that can be used, e.g., in surface-enhanced Raman spectroscopy (SERS), where 
typically the plasmon resonance of the system cannot be adjusted after fabrication 27,28 , or if they can be adjusted29,30, then the process is irreversible. Our DNA-nanoparticle bionanoactuator adds that functionality with a reversibly tunable surface plasmon resonance.

\section{Experimental}

The DNA strands (see supplementary information Table S3 for DNA sequences) were purchased from Integrated DNA technology (IDT, Coralville, lowa, USA). Neutral cysteine-tagged chimeric avidin was produced in Escherichia coli and purified using 2-iminobiotin affinity chromatography ${ }^{31}$. Bovine serum albumin (BSA), d-Biotin, poly(ethylene glycol) methyl ether thiol (SH-PEG, $M n=800)$, bis(p-sulfonatophenyl)phenylphoshine dehydrate dipotassium salt (BSPP) and AuNPs with $80 \mathrm{~nm}$ diameter (Lot\# MKBV4360V) were purchased from Sigma Aldrich (3050 Spruce st., St. Louis, M O 63103, USA). The UV-vis absorption of the AuNPs was measured using Perkin Elmer Lambda 850 UV-vis spectrometer. The ITO coverslip (8-12 $\Omega$ per sq, Lot\# 1200330) glasses were purchased from SPI supplies (West Chester, Pennsylvania, USA).

\section{Preparation of AuNPs coated with biotinylated DNAs}

For DNA-AuNP conjugation, we followed similar protocols as Storhoff and Mirkin with few minor adjustments ${ }^{32,33}$. For preconjugation, the spherical AuNPs were surface stabilized by treating them in BSPP solutions overnight ( $12 \mathrm{~h}$ ). First, $0.2 \mathrm{mg}$ of BSPP was weighted and $300 \mu$ l of plain AuNP solution (156 $\mathrm{pM}$ ) was used to dissolve it (BSPP concentration $=0.67 \mathrm{mg} \mathrm{ml}^{-}$ 1). Attachment of DNA onto AuNPs was done via thiolated 3'end of the ssDNA. Note, that the thiol was included in every DNA strand shown below even if it is not mentioned separately. The 5 '-end was modified with biotin or left blank depending on the case. Before conjugation, the ssDNA strands were treated with $1 \mathrm{mM}$ TCEP to cleave the disulfide bonds between thiolgroups. The DNA-TCEP solution was incubated for $1 \mathrm{~h}$ and then $38 \mu \mathrm{l}$ of the solution was added to a separate tube containing $300 \mu \mathrm{l}$ of the BSPP-treated AuNPs. The DNA-AuNP solution was mixed overnight after which we started to add salt buffer to detach the free-end of the DNA from the AuNP surface. Typical problem with large AuNPs $(>60 \mathrm{~nm})$ is that they are very unstable even in buffers with moderate ion concentration in contrast to small AuNPs, which are resistant against high ionic strength. For this reason, we adjusted the salt concentration slowly during 3-4 day period, until slight color change was observed. This was done by adding $2 \mu \mathrm{l}$ of $60 \mathrm{mM} \mathrm{NaCl}$ to the tube containing $338 \mu \mathrm{l}$ of the DNA-AuNP solution. After approximately $8 \mathrm{~h}$ another $2 \mu \mathrm{l}$ of $60 \mathrm{mM} \mathrm{NaCl}$ buffer was added, followed by overnight incubation. This was repeated until the color change was observed, with the final concentration of $2.1 \mathrm{mM} \mathrm{NaCl}$.

The mixtures were centrifuged ( $2 \mathrm{~min}, 2000 \mathrm{rcf}$ ) and the supernatant was removed. The volume was adjusted to $300 \mu \mathrm{l}$ by adding $0.1 \mathrm{mM}$ NaPhos $1 \mathrm{mM} \mathrm{NaCl}$ (NaPo-NaCl buffer, $\mathrm{pH}$ 7.6) and the centrifugation process was repeated 2 times. In the last run the volume was adjusted to $50-100 \mu \mathrm{l}$ with the same buffer. Finally, the UV-vis spectra and the hydrodynamic radius, $D$, were recorded from the final solution. The UV-vis spectra revealed, that the LSPR peak is roughly the same for all of the samples with or without DNA (see supplementary information Fig. S1), but the hydrodynamic radius differed as explained in the Results and discussion section.

For the calibration tests we utilized thiolated and biotinylated random ssDNA sequences with varying length, but for the final study a hairpin-DNA was utilized. The hairpin-DNA constitutes of a middle loop section and two arms ( 3 ' and 5 ' end) with different lengths. The loop has length of 25 bases and when folded forms $10 \mathrm{bp}$ zipper $\left(\mathrm{T}_{\mathrm{m}}=69^{\circ} \mathrm{C}\right.$, $\mathrm{M}$ fold $)$. The $5^{\prime}$ end arm has a length of 15 bases and the $3^{\prime}$ end arm has a length of 8 bases. To control the biotin density on the AuNPs, we made a solution $(98 \mu \mathrm{l})$ that contained biotinylated DNA (hairpin-DNA or sSDNA) and thiolated $3 \mathrm{~nm}$ long DNA strand with random sequence and without biotin (blocking-DNA). To test the optimal ratio the molar ratio of hairpin-DNA:blocking-DNA was altered from 1:0 to 1:999. Finally, the ratio 1:999 was chosen for all the experiments (ssDNA and hairpin-DNA) and, for comparing motion of individual particles under influence of electric field, we used AuNPs coated only with hairpin-DNA, as described in the Results and discussion.

\section{Protein and nanoparticle attachment to gold surface in SPR setup}

Bionavis SPR Navi 200 M P-SPR device and Bionavis thin glass substrates (Schott D263T glass slides) coated with gold layers were used to detect the resonance angle SPR (RA-SPR) signal resulting from the biomolecule and the gold particle binding. The experimental procedures were based on literatureestablished methods ${ }^{34}$. The sensors were fabricated by first cleaning the glass substrate using reactive ion etching (RIE) with O2 plasma ( $20 \mathrm{~W}, 15 \mathrm{~s}$ ), and then the sample was placed inside JYU self-made UHV e-beam evaporator. First, $2 \mathrm{~nm}$ adhesion layer of titanium was evaporated followed by evaporation of 42 or $48 \mathrm{~nm}$ gold layer.

In the SPR measurements, the duration of each protein, biomolecule or AuNP injection was 16 min. PBS was used as a running buffer and the total sample volume was $300 \mu \mathrm{l}$. The avidins were pre-treated with TCEP to reduce the amount of possible avidin dimers. Typical measurements started by mixing $6-10 \mu$ of chimeric avidin $\left(0.75 \mathrm{mg} \mathrm{ml}^{-1}\right)$ with $6 \mu$ of TCEP (1 $\mathrm{mM}$ ) and finally adjusting the volume to $300 \mu \mathrm{l}$ with PBS. This solution was incubated for $10 \mathrm{~min}$ at RT before injection. At the same time, base piranha solution was prepared by mixing $\mathrm{NH}_{4} \mathrm{OH}: \mathrm{H}_{2} \mathrm{O}_{2}: \mathrm{H}_{2} \mathrm{O}=1: 1: 5$, the solution was heated to $80^{\circ} \mathrm{C}$ and a gold sensor was placed in the piranha solution for $10 \mathrm{~min}^{35}$. After $10 \mathrm{~min}$, the sensor was immersed in water for short duration (5-10 s), then dried using $\mathrm{N}_{2}$ flow and lastly it was placed inside the SPR device (Navi 200 M P-SPR).

The sensor chamber was quickly flushed with PBS buffer and the chimeric avidin was injected in PBS. After roughly 16 min, the injection was stopped and the injection lines were quickly washed three times with $300 \mu$ l of the buffer. Next, $300 \mu$ of (0.29 mM) SH-PEG in PBS or $\left(500 \mu \mathrm{g} \mathrm{ml}^{-1}\right)$ BSA in PBS was 
injected into the chamber and after $16 \mathrm{~min}$ the previous purging procedure was repeated. Similar procedures were followed for biotin and AuNP injections. The biotin was injected in PBS and the AuNP were typically diluted 30-60 times in PBS to reduce the bulk effect. The tracked resonance angles were roughly at $65.96^{\circ}$ for $785 \mathrm{~nm}$ laser.

After the SPR measurements, the gold sensor was removed from the MP-SPR device, $\mathrm{ddH}_{2} \mathrm{O}$ was added to the area of the SPR chambers and a coverslip was placed on top of the sensor. The dark field (DF) images of the chambers were taken using Olympus microscopy equip with 50x DF objective (M PLANFL N) and Canon camera (EO5 D6, see supplementary information for more detail about the microscope setup).

\section{Assessment of the interaction between avidin and biotinylated- AuNP using DLS}

The biotin-avidin interaction was investigated by mixing the biotinylated-AuNPs with the chimeric avidin. In short, $1 \mu \mathrm{l}$ of chimeric avidin solution $\left(0.75 \mathrm{mg} \mathrm{ml}^{-1}\right)$ was added to $9 \mu \mathrm{l}$ of PBS and $0.5 \mu \mathrm{l}$ of TCEP and incubated for $10 \mathrm{~min}$. Then $2 \mu \mathrm{l}$ of this solution was added to $10 \mu \mathrm{l}$ of biotinylated-AuNP solution and the mixture was incubated for $10 \mathrm{~min}$. Finally, $12 \mu \mathrm{l}$ of the

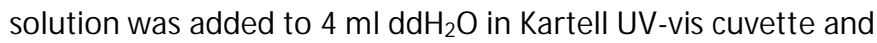
mixed thoroughly. The water was filtered beforehand using 0.1 $\mu \mathrm{m}$ pore size syringe filter and the cuvette was washed three times with the filtered water before use. The cuvette was placed inside DLS analyzer (BECKM AN Coulter N5 submicron Particle size Analyzer) and the mean hydrodynamic diameter and the polydispersity index were recorded. The measurement angle was $90^{\circ}$ in all of the measurements and the laser light source had wavelength of $632 \mathrm{~nm}$. For comparison, we also measured the hydrodynamic diameter of the original biotinylated-AuNP solutions and calculated a relative increase in the diameter after the avidin aggregation compared to the initial diameter.

\section{Preparation of the gold surface for immobilization of biotinylated AuNPs}

The Au-surface were prepared by cutting roughly $6 \mathrm{~mm} \times 6 \mathrm{~mm}$ boron-doped (100) silicon chips and cleaning the surface in a heated acetone bath followed by sonication in isopropanol bath for $30 \mathrm{~s}$. After this, the surface was still further cleaned using $\mathrm{O}_{2}$ plasma (Oxford Instruments / Plasmalab 80plus RIE: $\mathrm{O}_{2}$ flow 50 $\mathrm{sccm}, 60 \mathrm{~s}$ and $100 \mathrm{~W}$ ) and placed inside a vacuum evaporator. For making reliable electrical contacts between the front surface and the backside of the chip, the titanium adhesion layer and the subsequent gold layer were evaporated in three different angles: perpendicularly to the front surface, in 45 degree in respect to the backside surface and perpendicularly to the backside surface. For titanium, $2 \mathrm{~nm}$ layer was evaporated at each direction. For gold, $42 \mathrm{~nm}$ layer was evaporated in the front side and $10 \mathrm{~nm}$ for each angle in the backside.

\section{Immobilization of biotinylated AuNPs on gold surface coated with} chimeric avidins
The experimental setup is described in detail in the supplementary information, and a more general description is provided here. The nChiAvd-Cys solution was prepared by mixing $2 \mu \mathrm{l}$ of Cys-tagged charge-neutralized chimeric avidin $\left(0.75 \mathrm{mg} \mathrm{ml}^{-1}\right)$ to $1 \mu \mathrm{l}$ of TCEP $(1 \mathrm{mM})$ and $96 \mu \mathrm{l} \mathrm{PBS}$ and incubating the mixture for $10 \mathrm{~min}$ at room temperature. At the same time, the Au-surface was treated with similar basic piranha solution as in the SPR measurements $\left(\mathrm{NH}_{4} \mathrm{OH}: \mathrm{H}_{2} \mathrm{O}_{2}: \mathrm{H}_{2} \mathrm{O}\right.$ $=1: 1: 5)$ for $10 \mathrm{~min}$ at $80^{\circ} \mathrm{C}$. After the piranha treatment, the Ausurface was rinsed in $\mathrm{ddH}_{2} \mathrm{O}$ and the backside was dried using a clean room tissue. The chip was connected to self-made, polycarbonate holder (see supplementary information Fig. S5) by adding a small drop of conductive silver grease (OW Circuits works) to the gold layer on backside of the chip, circling the paste with varnish and gluing the chip to aluminium connections on the holder. After few minutes, $50 \mu \mathrm{l}$ of the avidin solution was added to the Au-surface and incubated for $30 \mathrm{~min}$. Then the surface was washed with PBS, substituted with $50 \mu$ of $1 \mathrm{mg} \mathrm{ml}^{-1} \mathrm{BSA}$ in phosphate buffered saline (PBS) and the solution was incubated for $30 \mathrm{~min}$. After this, the surface was washed with $\mathrm{NaPo}-\mathrm{NaCl}$ buffer.

In order to prepare the electric field apparatus, small layer of silver paste was added to the both contacts for ITO-coated cover glass on the holder and the ITO-coated cover glass was placed on top of the Au-surface. Then the cover slip was fixed using scotch tape and the resistance through ITO was measured to ensure good contact. Without the silver paste the contacts were typically poor. The input and output flow channels on the holder were pre-wetted for the AuNP injection, the holder with Au-surface was placed on microscope and ISMATEC tubing pump (ISM 597D) was inserted into the output channel. Image of the plain Au-surface was recorded for reference. Then, the extra water from the chamber containing the chip was removed and $50 \mu \mathrm{l}$ of the biotinylated AuNP (30-60 pM) in NaPo-NaCl buffer was injected into the chamber. The biotin-AuNP deposition was monitored for 15-90 minutes, until enough particles were immobilized on the surface but the density was still suitable for the LSPR scattering measurements (particle-toparticle distance larger than $10 \mu \mathrm{m}$ ). Then $1-2 \mathrm{ml}$ of $\mathrm{NaPo}-\mathrm{NaCl}$ buffer was run through the chamber until all of the free AuNPs were removed.

\section{Dark field measurement of the LSPR scattering spectra and optical imaging}

Here, we provide brief description of the dark field microscope setup and the LSPR spectra measurements, and more detailed description is provided in the supplementary information. The dark field microscope setup is illustrated in Fig. S6. The scattered light from individual gold nanoparticle was collected into the Acton SP2150 spectrograph equipped with Andor Ivac CCD camera via an optical fiber (core size $300 \mu \mathrm{m}$ ). To do this the tip of fiber was placed on the image plane of the output port of the microscope, and the part of the dark field image on the fiber spot was thus extracted to the spectrograph. The fiber tip could be visualized by light illumination from the spectrograph side, and individual particles could be selected for the LSPR 
measurement by moving the fiber spot on the particles using a XYZ-micrometer stage. The size of the fiber spot was roughly 10 $\mu \mathrm{m}$, and the deposition of biotin-AuNPs was stopped, before the average particle-to-particle distance became smaller than $10 \mu \mathrm{m}$ to enable individual particle measurements.

The LSPR measurements started by moving the fiber spot on the desired particle, waiting for $1 \mathrm{~min}$ and recording the initial, zero voltage spectra with 3-5 s integration time and averaging of 3 spectra. The following voltage sweeps consisted of a voltage change, 1 min stabilization time and recording the LSPR spectra with the same averaging and the integration time as before. Apart from changes in the transparency of ITO, we did not observe any changes in the background signal during the voltage sweeping, and for each particle, we recorded the background next to it on an empty spot. Canon EOS 6D camera was connected to the other output port of the microscope, and we used Canon EOS utility-software to remotely control the camera, to take dark field optical images and to monitor the sample surface during and after deposition of biotin-AuNPs. For the dark field images, the exposure time was 2.5 to 4 seconds.

\section{Measurement of the effect of the applied electric field}

For the large scale optical analysis, the voltage was swept from negative side to positive side (typically from $-3 \mathrm{~V}$ to $+3 \mathrm{~V}$ and back) to see the changes in the LSPR spectra of the surfacebound AuNPs. The voltage biasing is in respect to the gold surface. After each voltage step, the system was let to stabilize for $1 \mathrm{~min}$. The voltage was swept 3 times from positive side to negative side, and in each step an optical image was taken. Then, images were processed to see which particles changed the color notably (see supplementary information Fig. S7 and S8). The LSPR scattering spectra was measured for these particles. The microscope focus was checked and adjusted frequently during the measurements. Applying voltages higher than $\pm 3 \mathrm{~V}$ resulted in irreversible changes in the ITO due to the decomposition of $\mathrm{InO}_{2}$ and $\mathrm{SnO}_{2}{ }^{36}$, which eventually blocked the view to the Au-surface completely. Also, higher voltages caused bubbling in the system, and for these reasons, the voltage was kept as low as possible.

For the individual particle spectra analysis, depending on the amount of hairpin-DNA on AuNPs, the voltage was swept either from $-0.5 \mathrm{~V}$ to $+0.5 \mathrm{~V}$ with either 0.1 or 0.05 step $(0.1 \%$ ratio of hairpin-DNA) or from $-3 \mathrm{~V}$ to $+3 \mathrm{~V}$ with $0.5 \mathrm{~V}$ step (pure hairpinDNA). After each voltage step, system was left to equilibrate for $1 \mathrm{~min}$ and the LSPR scattering spectra was recorded. This was repeated for several voltage sweeps from negative to positive side. Both the individual scattering spectra of nanoparticle and the RGB data from optical images were analyzed to determine the individual and mass scale behavior of the biotinylatedAuNPs.

\section{Results and discussion}

Our electric field controllable DNA-AuNP-nanoactuator consists of a gold surface acting as an electrode, a DNA of interest attached to this surface from one end, and a negatively charged gold nanoparticle attached to the other end of the DNA. AuNP is used as an electric field controlled handle to stretch the DNA to change its conformation. The assembly of the nanoactuator is shown in Fig. 1a-c: (a) the binding of neutral cysteine-tagged chimeric avidins (nChiAvd-Cys) on the gold surface, (b) the passivation of the rest of the surface using a blocking agent (BSA or SH-PEG), and finally (c) the immobilization of $80 \mathrm{~nm}$ in diameter AuNPs, functionalized with biotinylated DNA (sSDNA or hairpin-DNA) and otherwise passivated with blocking-DNA. Initially, to test the immobilization, we used AuNPs coated with two different SSDNAs that either had both a thiol- and a biotinend (biotin-DNA) or just the thiol-end (blocking-DNA). Later on, to demonstrate the functionality of ready DNA-AuNPnanoactuators, we used thiolated and biotinylated SSDNAs with varying lengths ( $3 \mathrm{~nm}, 8 \mathrm{~nm}$ and $15 \mathrm{~nm}$, labelled DNA-s3nm, DNA-s8nm and DNA-s15nm). For general discussion, we will refer to all AuNPs coated with different biotinylated DNAs as biotin-AuNPs. The results of these tests were then applied to study the folding of a thiolated and biotinylated hairpin-DNA, where the opening and re-hybridization of the double stranded part adds additional entropy constrain leading to a switch-like behavior.

During the operation of the nanoactuator, the DNAs between the AuNP and the Au-surface are stretched by moving the charged AuNPs with electric field (Fig. 1e and 1f), while optically tracking the particle position by the change in its LSPR resonance, i.e., the scattering spectrum measured with dark field microscopy. The position of the particle can be further correlated to the conformation of the DNA as shown in Fig. 1e and $1 f$. We investigated the behavior of the nanoactuators both by monitoring individual particles as well as by analyzing an ensemble of particles. Also, for the hairpin-DNA, we studied the influence of the amount of biotin-avidin binding sites on the motion of the nanoactuator by varying the ratio between the 
hairpin-DNA and blocking-DNA attached to AuNPs (Fig. 1d and 1e).

\section{Assembly of the DNA-AuNP nanoactuator}

There are at least three major points to consider in the actuator design to obtain as freely moving AuNP as possible. First, the cysteine-tagged chimeric avidin was chosen as a platform for biotin targeted immobilization, since it allows a higher degree of control in the orientation of the avidin and in the motion of

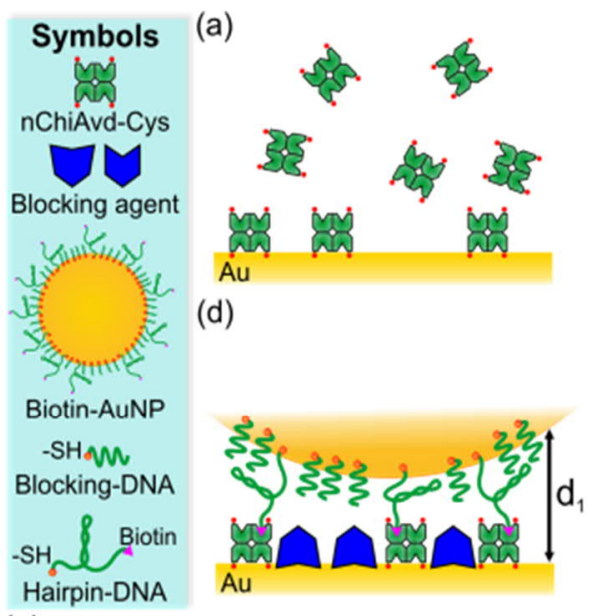

(g)

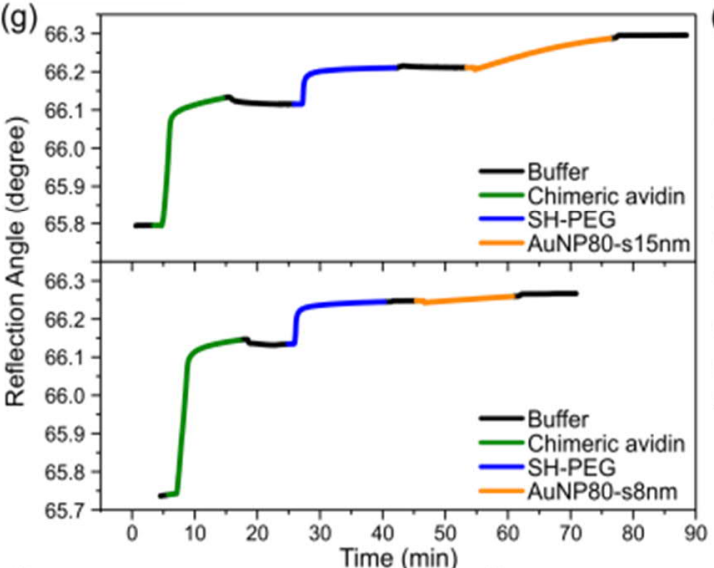

(b)

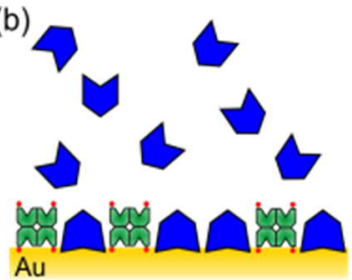

(e)

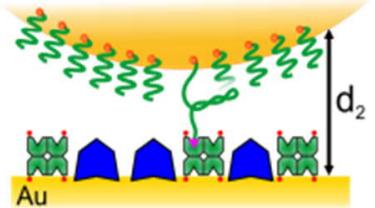

(c)

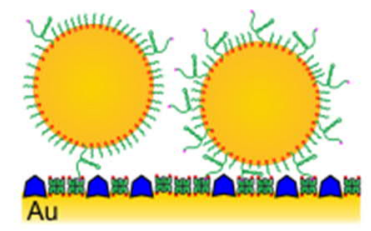

(f)

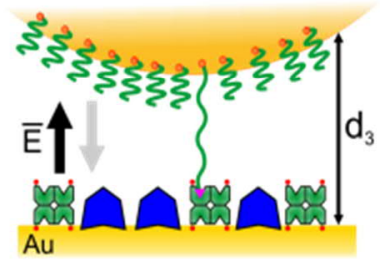

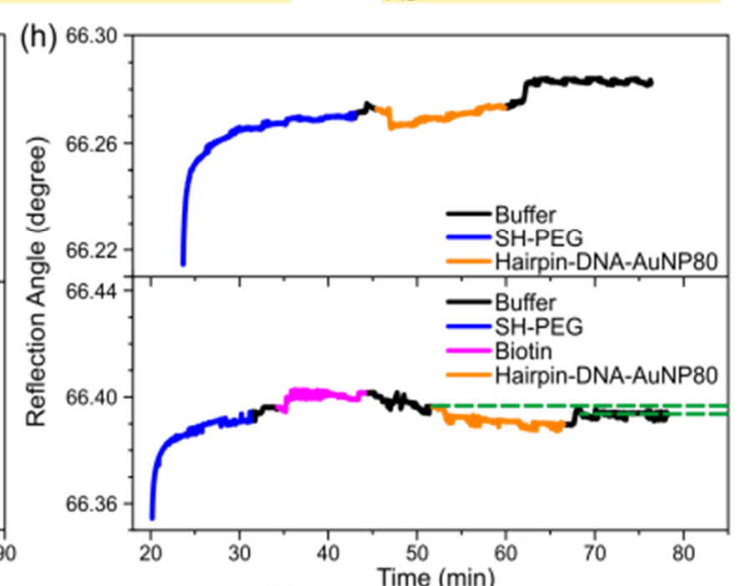

(i)
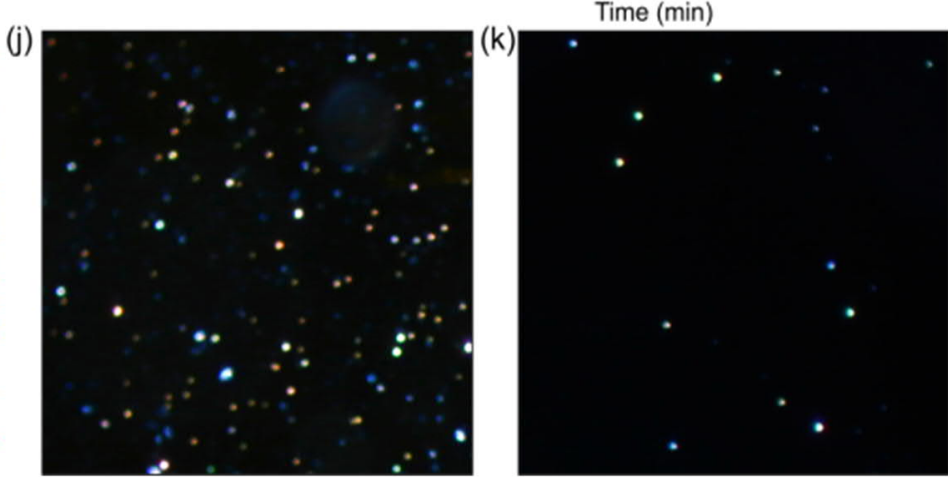

Fig. 1 Schematic illustration of the DNA-AuNP-nanoactuator and SPR characterization of its assembly. (a) Binding of neutral cysteine-tagged chimeric avidin (nChiAvd-Cys) on a gold surface, (b) the passivation of the remaining surface using a blocking agent (BSA or SH-PEG), (c) immobilization of AuNPs functionalized with biotinylated DNA. (d,e) After immobilization, the biotin-DNA (hairpin-DNA or plain ssDNA) is in a folded state and the particle is located close to the gold surface. The distance $d$ to the surface and the mobility of AuNP are influenced by the number of the linking DNAs, where more linking molecules indicate less mobility and thus shorter distance to the gold surface (distance $d_{1}$ versus $d_{2}$ ). (f) By applying an electric field the DNA hairpin can be opened (or SsDNA straightened) and the particle is pushed away from the surface $\left(d_{3}>\right.$ $\mathrm{d}_{2}$ ). When the electric field is switched off, the DNA loop can refold. (g) The SPR characterization of the assembly steps from (a) to (c) using AuNPs coated with $8 \mathrm{~nm}$ or $15 \mathrm{~nm}$ ssDNA linker. The binding of nChiAvd-Cys, blocking agent (SH-PEG) and biotin-AuNP are detected as positive shifts in the resonant angle. (h) Similar SPR characterization as in (g), but the specific avidin-biotin binding interaction is confirmed by passivating the surface bound avidins by free biotin before biotin-DNA attachment as a negative control (lower graph), and comparing it to the regular binding of the hairpin-DNA-AuNPs (upper graph). The green dashed lines in the lower graph indicate the slight negative shift for the binding of AuNP due to biotin passivation. (i-k) Dark field images of the surface after immobilization of the hairpin-DNA-AuNPs. (i) Surface after the process depicted in ac, (j) after the process involving additional passivation with free biotins as shown in lower graph of $(\mathrm{h})$, (k) a plain gold surface. Figure (i) shows a large amount of AuNPs with the same color, which can be attributed to the same distance from the surface and thus to specific binding. The AuNPs with varying colors $(j, k)$ indicate unspecific binding with varying distances. The widths of the images (i-k) are roughly $100 \mu \mathrm{m}$. 
the particle while still retaining a strong interaction with biotin $\left(K_{a}=10^{15} M^{-1}\right)^{37,38}$. Second, the blocking agent used to passivate the unoccupied surface after avidin immobilization is required, since the unspecific binding of biotin-AuNPs on the surface could greatly hinder the operation of the well-assembled actuators. Third, in addition to the length of the DNA linker, also the number of them connecting the individual AuNP and surface-immobilized avidin will set a limit on how far above the surface the particle can be lifted (Fig. 1d and 1e), as well as controls the force needed for particle displacement.

To address the two first points, and also, partially the third one, we used Resonance Angle Surface Plasmon Resonance (RA-SPR, Fig. $1 \mathrm{~g}, \mathrm{lh}$ and S4) to characterize the binding of the chimeric avidin (Fig. 1a), surface blocking agent (Fig. 1b) and the biotinAuNPs (Fig. 1c). In addition, immobilization of the biotin-AuNPs was also confirmed afterwards by using dark field microscopy (Fig. 1i-k).

The SPR measurements were carried out using standard procedures (see M ethods section) ${ }^{34}$. In each deposition step, a 16 min injection of the proteins or polymers to the sensor surface was followed by flushing of the measurement chamber using phosphate buffered saline (PBS) buffer, followed by next injection. The binding of avidin was detected as a positive shift in the SPR signal (Fig. 1g, green solid lines), which is expected for a material with non-negative, non-complex permittivity 39,40 . After 3.5 - 4.5 min injection, the binding curve started to reach a plateau, indicating that the surface becomes fully coated with avidins. The subsequent blocking agent (SH-PEG) injection followed a similar trend (Fig. 1g, blue lines), but the plateau was reached faster, since a large portion of the surface was already covered by the avidins, and the smaller blocking agents move more rapidly to the surface than the avidins.

To study the immobilization of biotin-AuNPs, we used hairpinDNA-AuNPs, AuNP-s8nm and AuNP-s15nm on avidinfunctionalized and passivated surface. These ssDNAs were chosen, since their lengths correspond to the hairpin-DNA in the folded and open states (see Methods section and Supplementary information for more details). The AuNP-s15nm had a higher positive SPR angle shift than the AuNP-s8nm as shown in Fig. 1g, because a different coupling between the LSPR of the AuNP and the Au-surface. Several publications have noted that the SPR signal depends strongly on the coupling between the AuNPs and the gold surface ${ }^{39,41}$ far away from the surface, AuNPs have only regular mass-contribution (positive shift). But, when AuNPs are close to the Au-surface, as in here, the SPR signal can have significant positive shift, negligible positive shift or even negative shift, depending on the change in the LSPR ${ }^{39}$. Here this means that for the biotin-AuNPs with longer DNA-linkers the mass-contribution is dominating and a SPR shift is noticeable, while for the shorter linkers, the SPR shift is negligible. For hairpin-DNA, we observed similar positive but small SPR shift as for the AuNP-s8nm, which indicates that the hairpin is mostly folded during the linking (Fig. 1h upper curve). To further clarify the situation, the immobilization of hairpinDNA-AuNPs was verified by dark field microscopy afterwards (Fig. 1i). As a negative control, we passivated the avidins on the surface with excess of free biotin after application of the blocking agent, and tested the hairpin-DNA-AuNP immobilization (Fig. 1h lower curve). In this case, no significant attachment of AuNP was observed in dark field microscopy (Fig. 1j) and the SPR shift was slightly negative (indicated by the green dashed lines in Fig. 1h), which could be attributed to a slight dissociation of avidins and blocking agent.

\section{Controlling the coverage of biotinylated DNA on AuNPs}

The AuNPs can be bound to the surface via one or several DNA strands (Fig. $1 \mathrm{~d}$ and 1e), and the number of bonds greatly influences the attachment and detachment properties of the particle 42 and, especially, the mobility of it. To achieve the highest degree of motion for the actuator, we minimized the amount of biotin-avidin bonds between a single particle and the surface. To achieve this, we fabricated AuNPs with different densities of biotins on the particle surface by mixing short blocking-DNAs without biotin with the longer biotinylated hairpin-DNA with molar ratios hairpin-DNA : blocking-DNA varying between 1:0 and 1:999 during the functionalization process and characterized the result using Dynamic Light Scattering (DLS). In this case, the hydrodynamic diameter, D, of the biotin-AuNPs should be decreasing as the amount of shorter blocking DNA is increased, which was also observed (Supplementary information Fig. S2). However, due to noisy result with lower amount of hairpin-DNA, we can only qualitatively correlate the hydrodynamic diameter to the amount of biotin groups on the surface of biotin-AuNPs.

For a better estimation of the amount of the biotinylated DNA, we used a fact that a typical density of thiolated DNA on the surface of AuNP is $0.08-0.16$ strands per $\mathrm{nm}^{2} .43$ The estimated total amount of DNA sequences on the surface of a single NP is then roughly 20000 , when taking into account the length of the DNA used and the size of the NP. Based on this and the ratio between the biotinylated hairpin-DNA and the non-biotinylated blocking-DNA in the solution, we can estimate the amount of hairpin-DNAs to be roughly 20 per particle in the case of $0.1 \%$ hairpin sample and about two in the lowest tested hairpin-DNA portion of $0.01 \%$.

Although the hydrodynamic diameter of the particle is related to the biotin density on the particle surface, it does not necessary correlate with the binding efficiency between biotinAuNP and avidins. To further characterize this and to verify our estimation of the amount of the biotinylated DNA per NP, the hairpin-DNA-AuNP was tested for its capability to cluster with avidins in solution (see Experimental section). It is expected that the average hydrodynamic diameter of the particle or particlecluster in the formed avidin-AuNP mixture would increase with the number of biotinylated DNAs at the surface of AuNP due to a higher probability of particle clustering. To analyze this, we calculated the relative increase in the hydrodynamic diameter $D_{\text {rel }}=\left(D_{\text {final }}-D_{\text {ini }}\right) / D_{\text {ini, }}$, where $D_{\text {final }}$ and $D_{\text {ini }}$ are the hydrodynamic diameters after and before the mixing of the biotin-AuNPs with avidin (Fig. 2a).

The same mixing ratios were used during the functionalization process. The relative increase in $D_{\text {rel }}$ and thus the amount of aggregation has a peak at ratio $1: 1$ and starts to decrease when 
increasing the amount of blocking-DNA as shown in Fig. 2b. This is due to the decrease in the number of the biotins and thus avidin binding points on the particle surface. On the other hand, the decrease in the apparent hydrodynamic radius observed when reducing the amount of blocking-DNA from 1:1 ratio, can be due to an insufficient avidin to biotin-AuNP ratio, or because too high surface density of biotin does not support efficient avidin-biotin binding ${ }^{44}$. Interestingly, we observed that AuNPs without any biotin, i.e., coated with only blocking-DNA, had the same $20 \%$ increase in the hydrodynamic diameter after mixing with avidin as compared to the control AuNPs without any DNA. This might be due to non-specific binding of avidin on AuNP or bulk effects. Yet, the hairpin-DNA samples with only $0.1 \%$ of biotinylated hairpin-DNA (ratio 1:999) showed still higher increase in hydrodynamic radius after addition of avidin (30\%) compared to the control sample (20\%). Assuming that the $0.1 \%$ hairpin-DNA sample does not form particle-particle dimers and that the $20 \%$ increase is due to the bulk effects, then the increase from $20 \%$ to $30 \%$ would roughly correspond to the size of addition of one avidin layer (see Supplementary information for more details).

The aggregation process was greatly diminished when lowering the amount of hairpin-DNA down to $0.1 \%$, and vanished totally with $0.01 \%$ concentration. This agrees very well with the above estimation, especially if taking into account that the hairpinDNA is longer than the blocking-DNA, and thus slower in diffusion, which most probably lowers its contribution within the attached DNA compared to the concentration ratio in solution. Since the aggregation process was significantly reduced but still happening at the 1:999 ratio, we used this ratio to fabricate all biotin-AuNPs (ssDNA: $3 \mathrm{~nm}, 8 \mathrm{~nm}$ and $15 \mathrm{~nm}$, and hairpin-DNA) for the electric field manipulation studies.

\section{Numerical calculation of the LSPR spectra of the DNA-AuNP- nanoactuator}

As mentioned above, we utilized the optical properties of the AuNP to track its position above the gold surface; the LSPR scattering spectra of AuNP is very sensitive to the distance between the nanoparticle and the surface. We used Finite Element method (FEM) (Comsol Multiphysics versio 5.1) to
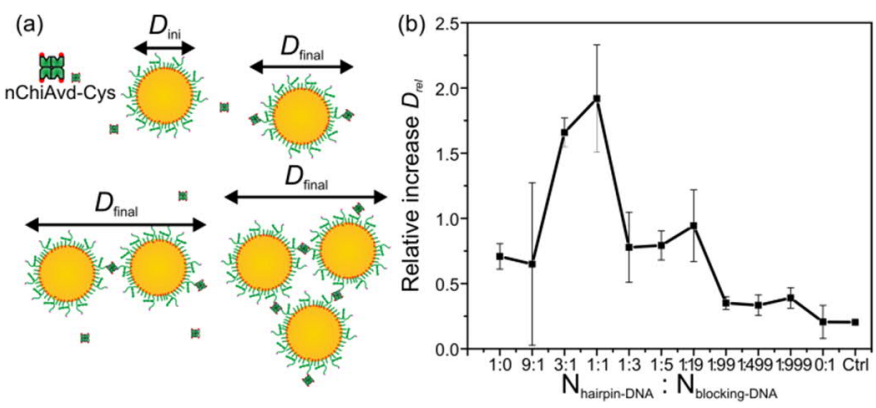

Fig. 2 Clustering of biotin-AuNPs with different ratio of hairpin-DNA and blocking-DNA in the presence of avidin. (a) A schematic view of the avidin-biotin-AuNP aggregation process. After mixing the biotin-AuNPs with chimeric avidin, the average size of the species increases due to the aggregation, which can be seen as an increased hydrodynamic diameter $D_{\text {final }}$ compared to the initial hydrodynamic diameter $D_{\text {ini. }}$ (b) The relative increase of the hydrodynamic diameter $D_{\text {rel }}$ versus hairpin-DNA:blocking-DNA ratio. Ctrl means a control AuNPs without any DNA. solve the LSPR spectra of the AuNP-Au-surface coupled system as a function of the distance $d$ (Fig. 3). The scattering spectra for transverse electric (TE) and transverse magnetic (TM ) polarized light in Fig. 3d-f show, that the main LSPR peak shifts from 570 $\mathrm{nm}$ to $660 \mathrm{~nm}$, as the distance $d$ decreases from $20 \mathrm{~nm}$ to $1 \mathrm{~nm}$. There is also a secondary peak appearing around $550 \mathrm{~nm}$, when the particle-to-surface distance is $5 \mathrm{~nm}$ or less. Similar behavior has been reported in the literature $22,28,45,46$, and it can be interpreted as an interaction between the real particle and a conduction surface-induced mirror particle. The main LSPR peak, which redshifts as the particle is brought closer to the surface, corresponds to the parallel dipole-dipole interaction between the real particle and the mirror particle. The secondary peak corresponds to in-plane dipole-dipole interaction. Both the in-plane and parallel dipole-dipole excitations create high electric fields that are confined within the gap between the particle and the surface (Fig. 3b, 3c and S14). Fig. 3f shows the LSPR scattering peak position dependency on the distance $d$ for both TE and TM polarized light ${ }^{47}$. Since we are probing with unpolarized light, the position of the AuNP is correlated to the average LSPR peak position of the two polarizations, which will be further correlated to the motion of the actuator and then to the stretching of the DNA.

\section{Manipulation of DNA-AuNP nanoactuators by electric field}

To implement all the above-mentioned findings for the functionality studies of ready DNA-AuNP-nanoactuators, we built an experimental setup, where we can observe nanoparticles on a gold surface within liquid environment by using in-situ dark field microscopy, while applying a voltage to create an electric field within the liquid medium. Moreover, liquids can be pumped in and out of the measurement flow

(a)
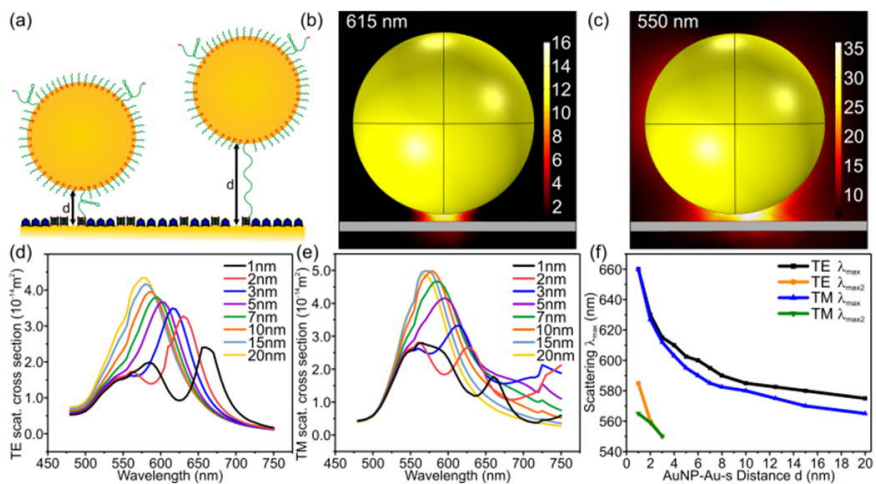

Fig. 3 Numerical simulation data of an $80-\mathrm{nm}$ gold nanoparticle coupled to the gold surface. (a) A schematic view of the simulated system. The distance $d$ between the particle and gold surface is varied from $1 \mathrm{~nm}$ to $20 \mathrm{~nm}$ and the scattering cross section of the TM and TE polarized excitation is calculated numerically. (b) The field enhancement of the AuNP-Au-surface system at $615 \mathrm{~nm}$ (TM polarization), when d is 3 $\mathrm{nm}$. The field is highly confined between the surface and the particle. (c) The field enhancement of the AuNP-Au-surface system at $550 \mathrm{~nm}$ (TM polarization), when $d$ is 3 $\mathrm{nm}$. The field is mostly confined to the gap but more spatially distributed as compared to system excited using longer wavelength (b). (d,e) The simulated TE and TM scattering cross-section of the AuNP-Au-surface system, when the distance is changed from $1 \mathrm{~nm}$ to $20 \mathrm{~nm}$. (f) The scattering cross-section wavelength maximum versus the distance $d$, where the LSPR scattering peak redshifts as the particle is brought closer to the Ausurface. There is also a secondary peak appearing when the particle-surface distance is exceeding $4 \mathrm{~nm}$, which can be seen in Fig. (d) and (e). 
chamber during the experiments to deliver studied assemblies as a suspension or to change the buffer conditions, e.g., $\mathrm{pH}$, amount of salts or temperature. This liquid flow holder was designed so that the chip with the active Au-surface could be fixed on the bottom and the chamber can be sealed using an ITO glass on top. The holder contains electric contacts for both the ITO and the Au-surface for applying the electric field. See supplementary information for more details about the setup.

The fabrication of the DNA-AuNP-nanoactuators was carried out similarly as in the RA-SPR measurements above (Supplementary information Fig. S5): After a hydrophilic treatment, the gold-coated Si chip was glued into the contact of the holder using silver paste and varnish. The resistance between outside contacts and the Au-surface was measured to ensure good electrical contacts $(R \sim 10-250 \Omega)$. The avidin and BSA solutions were prepared similarly as in the SPR measurement, but the solution was incubated on the Ausurface instead of injection. The avidin solution was first applied on the surface for $30 \mathrm{~min}$, followed by a washing step using PBS buffer and a subsequent incubation of BSA for $30 \mathrm{~min}$. After protein deposition, the sample was cleaned by injecting $50 \mu \mathrm{l}$ of $0.1 \mathrm{mM}$ NaPhos $1 \mathrm{mM} \mathrm{NaCl}$ buffer (pH 7.6, NaPo-NaCl) and the chamber was sealed using ITO coated glass by connecting two opposite sides of the ITO glass to upper contacts in the holder. Again, the resistance from external contact to the floating side of the ITO was measured to ensure good contacts $(R \sim 8-50 \Omega)$. The input and output fluid channels of the holder were prewetted using $\mathrm{NaPo}-\mathrm{NaCl}$ and the holder was placed under optical microscope. Typical immobilization of the biotin-AuNPs consisted of pumping of the biotin-AuNP solution into the chamber, waiting 15 to $90 \mathrm{~min}$ for biotin-AuNPs to immobilize and the subsequent flushing of the non-bound biotin-AuNPs using $\mathrm{NaPo}-\mathrm{NaCl}$.

The nanoactuator motion was studied both statistically in largescale and by monitoring individual actuators. The individual actuators were studied by recording the LSPR scattering spectra of a single particle with dark field microscopy while applying an electric field. However, this scheme could not be scaled up due to the serial nature of the measurements. Instead, we used a camera to take optical dark field images containing a large ensemble of actuators and analyzed the color (wavelength) changes of the particles via the RGB data. The large-scale behavior was analyzed to evaluate the percentage of controllable actuators, i.e. the fabrication yield, and to observe the collective motion of the system, which gives insight of the folding properties of the biomolecule. On the other hand, the individual measurements can be used to directly evaluate the optical properties of the system, i.e., the LSPR peak position and how accurately it can be controlled, which is important when considering application in, e.g., SERS or fluorescence enhancement.

The large-scale measurements were performed by sweeping the bias voltage from negative to positive voltages in constant steps (see Methods section). After each voltage change and subsequent 1 min stabilization, an optical image of the sample was recorded. After the measurements, the optical images were processed using the image-J program to extract the RGB data of the biotin-AuNPs in respect to the bias voltage (see Supplementary information). The RGB data was further converted into the corresponding hue, saturation and value (HSV) numbers (see Supplementary information). The HSV representation was selected, since the visible spectra can be represented as different hue values from $0^{\circ}$ to $360^{\circ}$ in a convenient linear scale. The issue here is that the hue values cannot be directly related to the maximum wavelength of the LSPR scattering spectra and thus to the position of the AuNP. To overcome this, we calibrated the system by measuring both the LSPR spectra and the corresponding RGB (HSV) values from several AuNPs without applied electric field (see Supplementary information). The hue versus LSPR peak wavelength was plotted for calibration, and, in particular, we estimated the shift in hue value corresponding to particle movement from the close vicinity of the surface (distance $5 \mathrm{~nm}$ or less in Fig. 3) to position distal to the surface (distance $\sim 20 \mathrm{~nm}$ in Fig. 3 and S9, see supplementary information for more details). The distance of 5 $\mathrm{nm}$ was selected, since this would correspond to the thickness of the chimeric avidin and the passivation layer, and most probably the particle would not penetrate this layer. A minimum shift in hue for a significant movement was found out to be around $40^{\circ}$, and for any further processing, we selected only those particles that had at least once a Hue shift of $40^{\circ}$ or more, when the direction of the voltage was switched several times.

\section{Demonstration of the nanoactuator operation by SSDNA}

To demonstrate the controllable behavior, we first analyzed nanoactuators assembled with different ssDNAs of varying lengths, i.e., DNA-s3nm, DNA-s8nm and DNA-s15nm. As described above, we analyzed the behavior of each particle under electric field from the optical images to evaluate the degree of its controllability. Most of the particles that had at least $40^{\circ}$ shift in the hue, could be categorized roughly into four groups (Fig. 4 and S10): the particles which could be pushed away with negative E-field and pulled towards the gold surface with positive E-field (group 1, orange histograms, 4.6-8.9\% of AuNPs); particles which had opposite behavior compared to the group 1 (group 2, blue histograms, 0.2-5.2\%); particles which eventually stuck far away from the surface (group 3, purple histograms, 1.8-3.8\%) and particles which eventually got stuck close to the surface (group 4, green histograms, 0.4-2.1\%). The black histograms of the last column illustrate the data measured for all particles, i.e., all the groups together.

The difference between the group 1 and group 2 can be at least partially attributed to the charge distribution of the particles. We characterized the Zeta-potential of the different biotinAuNPs, which showed that most of the samples had a large portion of negative charged particles (94.2-99.1\%) and a small portion of positively charged particles (0.9-5.8\%), which would indicate that negative particles contribute to the group 1 and positive particles to the group 2 (see Supplementary information Fig. S3 and Table S2). Also, the surface charge and double-layer induced by the electric field can affect the behavior of the AuNPs. Overall, the interaction of AuNPs and 
different charges in the system leaves a lot of room for interpretation and further studies would be required to fully assess the situation.

Fig. 4a shows the results of the analysis for the AuNP-s15nm sample. For the particles of the group 1 ( 56 pcs, orange) the hue (H) values form a Gaussian distribution which shifts continuously to higher values with negative voltage and are pulled back towards $0^{\circ}$ with positive voltage. We also observed similar behavior for particles with $8 \mathrm{~nm}$ spacers (see Supplementary information), but the AuNP-s3nm sample had particles located closer to the surface due to the shorter linker and the shifts in the histogram position were negligible (Fig. S10). For AuNP-s8nm, we observed a number of particles belonging to the group 2, but only few were observed for sample $\mathrm{s} 15 \mathrm{~nm}$. As discussed above, this can be due to varying charge of the functionalized gold particles in the s8nm, while in s15nm samples the longer DNA guarantees negative charge. This is further verified by the high number of $s 15 \mathrm{~nm}$ particles in the group 3 compared to the group 4 (Fig. 4a).

Overall, it is evident that in many cases the particles are moving initially with the electric field, but eventually stop responding (Fig. 4, S10b and S13, the groups 3 and 4). Even in the case of the AuNP-s8nm, the collective motion of the particle seems to have continuous flow with the shape of Gaussian distribution as in the AuNP-s15nm sample, which would be expected for a nanoactuator anchored via a string with thermal fluctuations. However, the behavior degrades with increasing number of voltage switches. The sensitivity of the nanoactuator behavior on a successful linking is well demonstrated by the fact that most of the particles are unmovable and located near the surface (Fig. S11). For this reason, the chosen threshold for analysis, i.e., the minimum of $40^{\circ}$ shift in the $\mathrm{H}$ value, was indeed necessary. Here it should be emphasized, that the histograms illustrate overall collective motions of the particles, which have similar behavior at some point during the measurements.

\section{Applying the nanoactuator to study the hairpin-DNA}

Finally, we utilized the DNA-AuNP-nanoactuator to study the conformation of the hairpin-DNA. The overall results resemble greatly the above case of plain ssDNA. However, even though the particles have a specific behavior under the electric field, allowing also the division to the four groups, the motion of the hairpin-DNA-AuNPs seems to fluctuate mostly between two distinct values (dotted and dashed vertical lines in Fig. 4b) as the voltage is swept. It is clear from the simulations and $H, \lambda$ calibration, that those values correspond to the situations where the particle is close to the surface and far away from the surface. As seen in the RA-SPR measurements, without any electric field the hairpin-AuNP is close to the surface, i.e., the hairpin-DNA is in a folded state as shown in Fig. 4c. Applying high enough electric field leads to opening of the hairpin by straightening the DNA-strands (Fig. $4 \mathrm{~d}$ and $4 \mathrm{e}$ ). But when pulling the particle back to the surface, the hairpin can relax and folds back (Fig. 4f), and thus we mainly observe quantized fluctuations of the particle position. On the other hand, because we always see particles with both positions, it could be that thermally driven hybridizations and openings of the hairpin happens all the time and we only affect the balance between these two states by applying the electric field.

Table 1. The assessment of controllability of the DNA-AuNP-nanoactuator

\begin{tabular}{ccc} 
Sample & $\begin{array}{c}\text { Fraction in the group 1 } \\
(\%)\end{array}$ & $\begin{array}{c}\text { Fraction in the group 2 } \\
(\%)\end{array}$ \\
\hline hairpin-DNA & 6.6 & 5.2 \\
\hline DNA-s15nm & 4.6 & 0.2 \\
\hline DNA-s8nm & 8.9 & 8.1 \\
\hline
\end{tabular}

The controllability of the nanoactuator for both the hairpin-DNA and the ssDNAs were assessed based on the percentage of particles in the groups showing systematic behavior under an electric field, i.e., the group 1 and group 2 (Table 1) compared to all of the immobilized particles (no threshold for $\mathrm{H}$ value). If combining the two groups, the percentage of controllable particles was about $12 \%$ for the hairpin-DNA and 4.6 to $17 \%$ in the case of the ssDNA samples. The overall RGB data of all the immobilized particles for all of the samples in Fig. S11 shows that most of the particles are located near the surface $\left(\mathrm{H} \approx 0^{\circ}\right)$ during the measurements, which suggest that the particles, even after the minimization of the number of biotins, are still bound to the surface too strongly to be displaced by electric field.

To further test the possible enhancing effect of even more reduced densities of avidin and biotin, we fabricated samples with only about one or two avidin within the area covered by a single AuNP, to prevent binding via multiple avidin-biotin links. These tests confirm the above deduction, that non-specific binding is the most significant factor lowering the yield of the nanoactuator assembly (see Supplementary information). Lowering the avidin density makes the formation of proper avidin-biotin binding slower and thus the non-specific binding has more time to happen during the AuNP deposition. Thus, with the lowest avidin density there appears to be almost only non-specifically bound particles. On the other hand, the reduction of the biotinylated hairpin-DNA to $0.01 \%$ did not produce enough binding ability, as was noticed also in the aggregation tests (discussed above), and virtually all the particles were non-specifically bound. This also indicates that most of the non-functional particles correspond to the nonspecifically bound particles. 

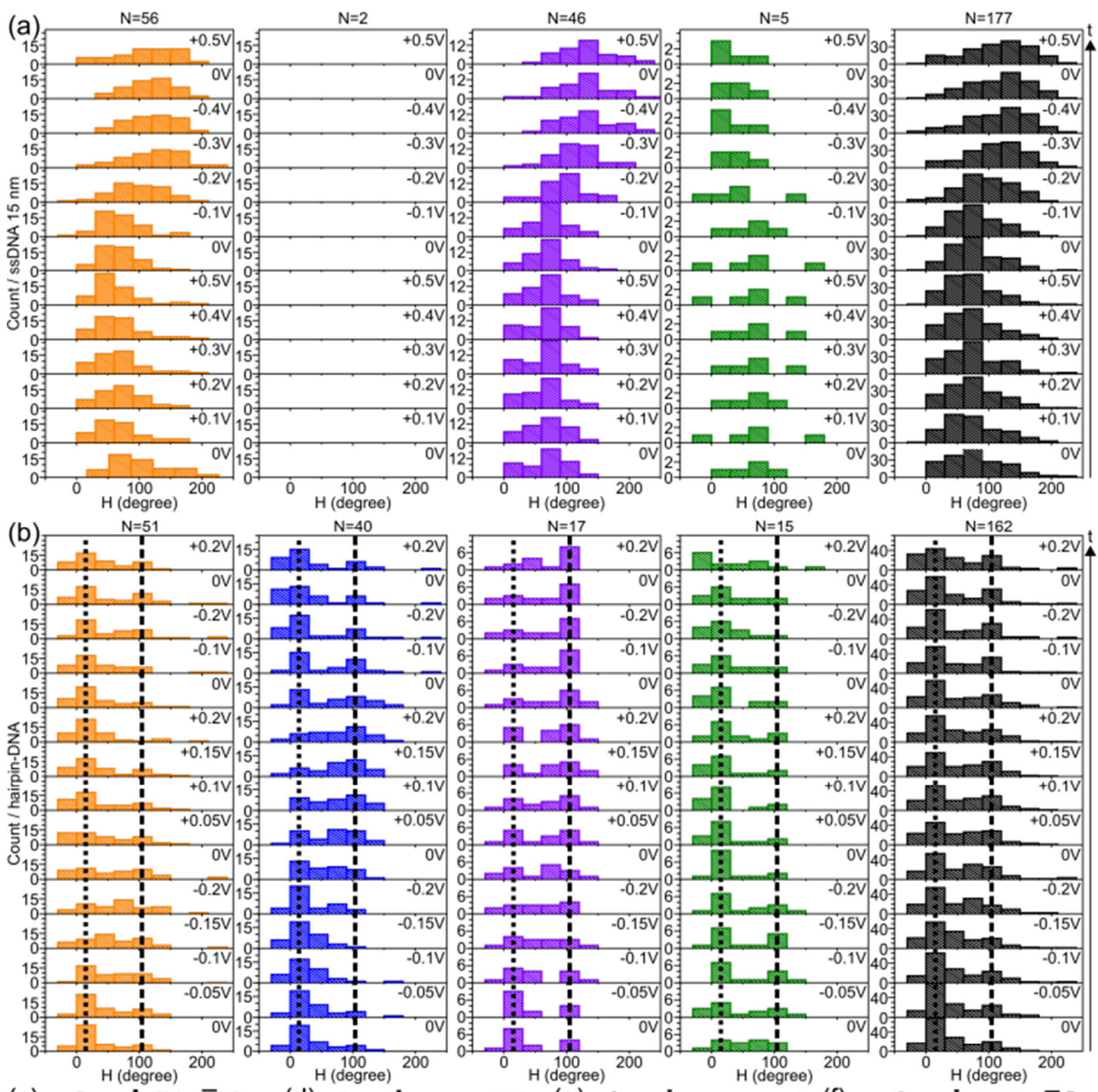

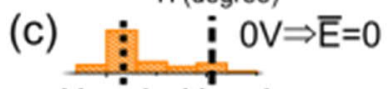

$\mathrm{H}_{1} \rightarrow \mathrm{d}_{1} \mathrm{H}_{2} \rightarrow \mathrm{d}_{2}$

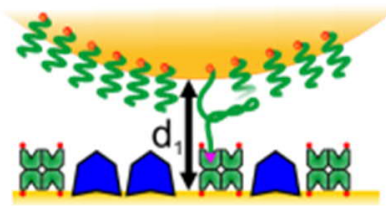

(d)
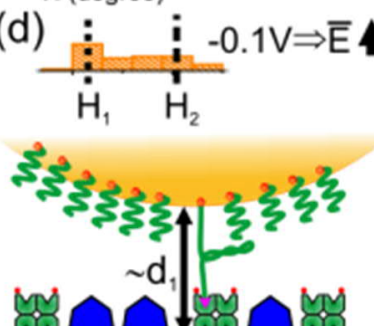

(e)

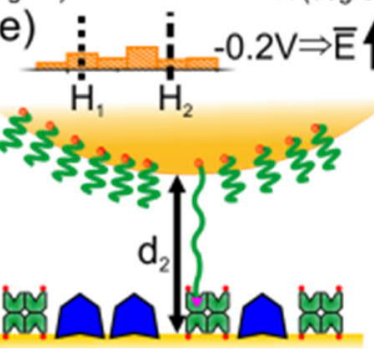

(f)

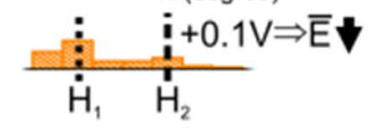

Fig. 4 The histogram data of the color changes of AuNPs bound to avidin-coated gold surface via biotinylated DNAs. (a) The histogram data of AuNPs conjugated with $0.1 \%$ of biotinylated $15 \mathrm{~nm}$ spacer DNA and passivating DNA. The numbers above the histograms indicate the number of analyzed particles. The orange histograms show particles that move away from the surface with negative electric field. The blue histograms represent a group of particles that behave opposite to particles in the orange histograms. The violet histograms show particles that slowly move away from the surface and the green histograms show particles that are stuck close to the surface. The black histograms show data of all of the analyzed particles. (b) Similar histogram data as in (a), but for AuNPs with $0.1 \%$ of hairpin-DNA and blocking DNA. (c)-(f) Schematic illustration of opening and folding of the hairpin-DNA, where the hairpin is initially folded when no external electric field is applied. The histogram data in panels $(c-f)$ depicts the first $(\mathrm{V}=0 \mathrm{~V})$, third $(\mathrm{V}=-0.1 \mathrm{~V})$, fifth $(\mathrm{V}=-0.2 \mathrm{~V})$ and eighth $(\mathrm{V}=+0.1 \mathrm{~V})$ histogram starting from bottom in the panel (b). When only a small electric field $\bar{E}$ is applied, the hairpin stays closed and the particle is located close to the surface ( $d \approx d_{1} \rightarrow H_{1}$, indicated by the dotted line). By applying high enough field $\bar{E}$, the hairpin can be opened and the particle is pushed far away from the surface ( $d=d_{2} \rightarrow H_{2}$, indicated by the dashed line). When the particle is pulled towards the surface and let to relax ( $\approx \approx d_{1}$ ), the hairpin can fold back. 


\section{Study of individual hairpin-DNA-AuNP nanoctuators}

Selected hairpin-DNA-AuNPs, which seemed to be moving freely in the large-scale measurements, were subjected for the individual particle measurements. The voltage-dependent LSPR scattering spectra were measured for several movable AuNPs (Fig. 5, S12 and S13). Fig. 5a and 5b show spectra of Particle A and Particle $B$ with $0.1 \%$ hairpin-DNA, respectively, whereas the particles $C$ and $D$ in Fig. $5 c$ and $5 d$ have the hairpin-DNA covering the whole particle. As the voltage was swept, it was typically observed that pure hairpin-DNA particles required $\pm 3 \vee(\bar{E} \approx$ $3.5 \cdot 10^{4} \mathrm{~V} \mathrm{~m}^{-1}$ ) to move, while particles with $0.1 \%$ hairpin-DNA needed only $\pm 0.5 \mathrm{~V}\left(\bar{E} \approx 0.6 \cdot 10^{4} \mathrm{~V} \mathrm{~m}^{-1}\right)$. This observation is reasonable, since AuNPs with higher amount of biotin-DNA likely form more bonds with the avidin-coated surface and consequently, there are more connecting DNA hairpins to resist the stretching movement. The LSPR scattering spectra changes can be also seen as color changes in the dark field images, as shown in Fig. $5 e$ (negative voltage) and $5 f$ (positive voltage) for particle $A$ and for particle $B$ in Fig. $5 g$ (negative voltage) and $5 \mathrm{~h}$ (positive voltage).

Interestingly, the main LSPR peak position for particle A and B are typically around 567-585 $\mathrm{nm}$ when they are pushed away from the surface by negative field and the peak shifts to 600 $640 \mathrm{~nm}$ when pulled by positive voltage. Based on the FEM simulations (see Fig. 3f), these peaks would correspond to distances $18 \mathrm{~nm}$ ( $570 \mathrm{~nm}$ peak) and 2-5 nm ( $620 \mathrm{~nm}$ peak) above the Au-surface. However, there are also peaks at $535 \mathrm{~nm}$ and $660 \mathrm{~nm}$ as shown in Fig. 5a and $5 \mathrm{~b}$ (blue dot-dashed lines and orange dashed), when the particle is pushed with negative voltage, but, based on the FEM-simulations, these peaks should
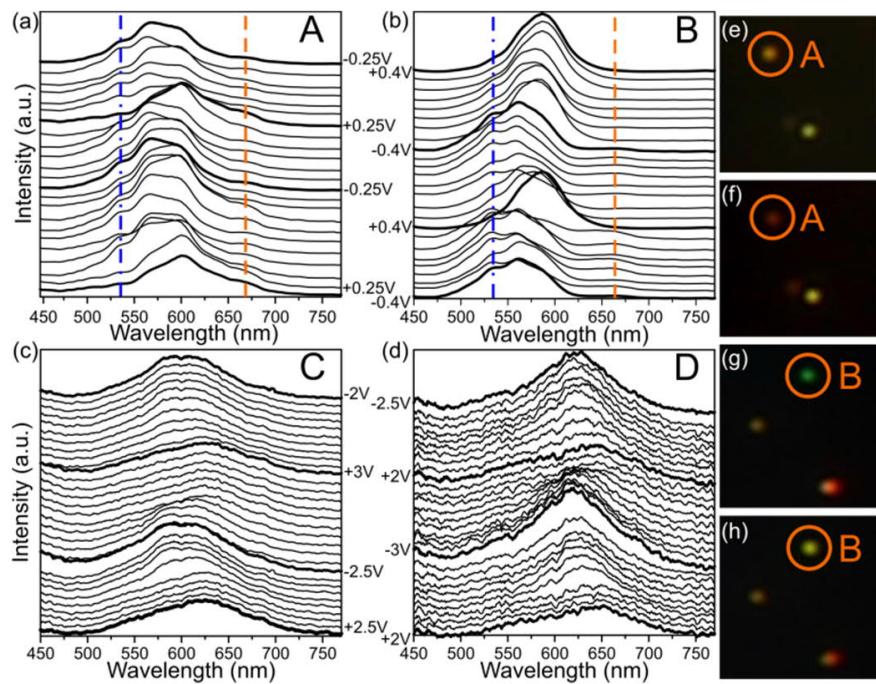

Fig. 5 The LSPR scattering spectra of individual AuNPs bound to the avidin-coated Ausurface via biotinylated hairpin-DNAs. (a-d) The LSPR spectra of particles A-D. Particles A and $B$ are coated with a mixture of $0.1 \%$ biotin-DNA and $99.9 \%$ blocking-DNA, while $C$ and $D$ are coated with biotin-DNA alone. The secondary peaks at $535 \mathrm{~nm}$ (blue dash-dot line) and $660 \mathrm{~nm}$ (orange dashed line) in Figures (a) and (b) are associated to particle position close to surface, and they are missing in Figures $(c)$ and $(d) .(e, f)$ Dark field images of the particle $A$ under either negative (e) or positive (f) electric field in respect to the gold substrate. $(\mathrm{g}, \mathrm{h})$ Dark field images of the particle B under either negative $(\mathrm{g})$ or positive (h) electric field in respect to the gold substrate. only be present when the particle is very close to the Ausurface. This indicates that despite the surface passivation, AuNPs still have a possibility to push themselves close to surface between the passivating molecules.

Since the exposure time is relatively long ( $3 \mathrm{~s}$ ) and while pushing AuNP away with negative voltage, both the close proximity peaks ( $530 \mathrm{~nm}$ and $660 \mathrm{~nm}$ ) and the far away peak $(580 \mathrm{~nm})$ are present (still the $580 \mathrm{~nm}$ peak being the largest), we probably see an average position of the particle $A$ and $B$ in respect to the Au-surface. Seeing this kind of combined spectrum for a single AuNP indicates that the particles are constantly moving up and down in relation to the surface. However, since the observed spectrum is clearly a sum of mainly two particle positions (close proximity and far away), instead of combination of spectra of all the possible positions, we can deduce that these two positions are the most pronounced ones. This agrees well with above deduction of constant thermally driven hybridization and opening of the hairpin. For some particles, we predominantly only see the $530 \mathrm{~nm}$ and the $660 \mathrm{~nm}$ peaks indicating that they are stuck in the vicinity of the surface (Fig. S12C).

The particles $C$ and $D$ with higher amount of biotin did not have similar behavior, but they rather had rigid and better-defined motion of the LSPR peak without fluctuations. The peak positions for particles $C$ and $D$ are moving between $590 \mathrm{~nm}$ and $623 \mathrm{~nm}$ and between $620 \mathrm{~nm}$ and $643 \mathrm{~nm}$, respectively, when pushed away and pulled towards the surface. For particle $C$, this would correspond to a motion, where a particle is stretched 7 $\mathrm{nm}$ and then brought back to a distance of $2.5 \mathrm{~nm}$, which is a bit less than the height of the chimeric avidin. For particle $D$, the motion would be between $3 \mathrm{~nm}$ and $1 \mathrm{~nm}$. These results have several consequences; on the one hand, it is vital to minimize the amount of avidin and passivating the surface, if one wants to achieve full particle motion and the best sensitivity. Increasing the amount of biotin on the surface of AuNPs would mean rigid, well-defined motion, which could be desirable for actuator applications.

\section{Conclusions}

In summary, we have fabricated and characterized a DNA and AuNP based nanoactuator utilizing particle-substrate distance dependent plasmonic coupling, and used this nanoactuator for molecular studies under applied electric field. First, the functionalization of AuNP with different biotinylated DNA strands were carried out using established protocols. We minimized the amount of biotin groups on the AuNP surface by mixing the biotinylated-DNA and a blocking-DNA in a very low ratio of 1:999, which was still enough to cause significant affinity between avidin and biotin-AuNPs as shown by the DLS analysis. We demonstrated using RA-SPR and dark field microscopy that these biotinylated AuNPs can be immobilized selectively to a gold surface functionalized with chimeric avidin and passivated with thiolated PEG or BSA. However, in SPR experiments, the biotin-AuNP binding to the chimeric avidin was not straightforward to show, since the SPR signal shift was typically weak, most probably due to the coupling between the laser- 
excited surface plasmon polaritons and the LSPR of the gold nanoparticles.

The nanoactuator scheme was used to analyze the motion and dynamics of the hairpin-DNA in respect to the ssDNAs with varying lengths under the influence of the electric field. It was discovered that the ssDNA samples had continuous flow on and off the surface, whereas the hairpin-DNA had a more quantized motion, which is the result of opening and folding of the hairpinDNA. When analyzing the behavior of the ssDNAs and hairpinDNA under the E-field, roughly $14-29 \%$ of the bound particles did change the color during the measurements at least once, and overall $5-17 \%$ of the bound particles could be controlled for an extended period. This indicates that even though the number of biotins on AuNPs was optimized, the majority of biotin-AuNPs have still too high affinity towards the avidins on the surface or are bound only non-specifically. To improve the former case, we reduced the density of avidin on the surface and the amount of the biotin-DNAs on the AuNP. It was observed that the reduction of either component can be done only to a certain extent, since the immobilization time of biotinAuNP increases significantly, which shows as increased number of non-specifically bound AuNPs. This indicates that the nonspecific binding is the most significant factor lowering the yield of the nanoactuator assembly, and that most of the nonfunctional particles are not properly assembled actuators, but only non-specifically surface-attached particles, which can be excluded from the analysis.

We were also able to characterize the movement of individual biotinylated hairpin-DNA-AuNPs under electric field manipulation. These studies reveal that the individual particles can have different behavior under E-field, most probably depending on the amount of attachments points between the Au-surface coated with avidins and the particle containing biotinylated DNA. Low amount of biotins resulted in a situation, where particle's LSPR peak could be moved freely between 565 $\mathrm{nm}$ and $640 \mathrm{~nm}$, which corresponds to the situations where hairpin-DNA is fully stretched or the particle is touching the chimeric avidin layer, respectively. Notably, the avidin layer (theoretical thickness of $\sim 3-5 \mathrm{~nm}$ ) may somewhat limit the sensitivity, which significantly increases, when the particle is closer than $5 \mathrm{~nm}$ as shown in the simulations. For optimal system, the avidin layer and the surface passivation could be further tuned, so that the particle would be capable to get closer to the sample surface but still not to get stuck on it. Some kind of nanopatterned surface could be of value here.

Also, when the biotin amount was lowered, position of the particles seemed to fluctuate on and off the surface when pushed away with electric field. Most probably, this was due to thermal motion. When there were higher amount of biotins per particle, the particles had more rigid motion with no secondary LSPR peak, meaning that the particles firmly attached to the gold surface. These together with the fact that the higher biotinylation density required larger voltages to see any shifts hints that the controlling the amount of biotins on AuNP surface is a key factor to enable free motion of the AuNP under influence of the electric field. Based on these results we can conclude that DNA-AuNP system offer promising platform for reversible E-field controlled nanoscale molecular actuator.

\section{Conflicts of interest}

There are no conflicts to declare.

\section{Acknowledgements}

This work was financially supported by Academy of Finland through projects 263526, 130900, 283011 for J.J.T., 263540 and 290506 for V.P.H and 263523 of M.A. K.T. received funding from Finnish Cultural Foundation. We thank Bionavis for equipment and essential expertise in the SPR analysis and Stephan Block (Freie Universität Berlin, Germany) for fruitful discussions and advices.

\section{Notes and references}

1 B. V. Dorst, J. M ehta, K. Bekaert, E. Rouah-M artin, W. D. Coen, P. Dubruel, R. Blust, J. Robbens, Biosens. Bioelectron. 2010, 26, 1178. Citations should appear here in the format $A$. Name, B. Name and C. Name, Journal Title, 2000, 35, 3523;

2 A. S. Ansari, Q. Husain, Biotechnol. Adv. 2012, 30, 512.

3 B. Pérez-López, A. M erkoçi, Trends Food Sci. Technol. 2011, 22, 625.

4 K.-I. Chen, B.-R. Li, Y.-T. Chen, Nano Today 2011, 6, 131.

5 M. Pilo-Pais, A. Watson, S. Demers, T. H. LaBean, G. Finkelstein, Nano Lett. 2014, 14, 2099.

6 J. W. Naughton, M. Sheplak, Prog. Aerosp. Sci. 2002, 38, 515.

7 K. Fu, S. Chen, J. Zhao, B. G. Willis, ACS Sens. 2016, 1, 444.

8 Y. Ke, T. Meyer, W. M. Shih, G. Bellot, Nat. Commun. 2016, 7 10935.

9 H. Gu, W. Yang, N. C. Seeman, J. Am. Chem. Soc. 2010, 132, 4352.

10 R. Elghanian, J. J. Storhoff, R. C. Mucic, R. L. Letsinger, C. A. M irkin, Science 1997, 277, 1078.

11 S. Block, B. J. Fast, A. Lundgren, V. P. Zhdanov, F. Höök, Nat. Commun. 2016, 7, 12956.

12 M. Banchelli, S. Nappini, C. M ontis, M. Bonini, P. Canton, D. Berti, P. Baglioni, Phys. Chem. Chem. Phys. 2014, 16, 10023.

13 C. Jiang, M. E. M cConney, S. Singamanemi, E. M errick, Y. Chen, J. Zhao, L. Zhang, V. V. Tsukruk, Chem. Mater. 2006, 18, 2632.

14 K. Tapio, J. Leppiniemi, B. Shen, V. P. Hytönen, W. Fritzsche, J. J. Toppari, Nano Lett. 2016, 16, 6780.

15 A. V. Pinheiro, D. Han, W. M. Shih, H. Yan, Nat. Nanotechnol. 2011, 6, 763.

16 S. R. Paludan, A. G. Bowie, Immunity 2013, 38, 870.

17 M. Zahid, B. Kim, R. Hussain, R. Amin, S. H. Park, Nanoscale Res. Lett. 2013, 8, 119.

18 K. E. Dunn, M. A. Trefzer, S. Johnson, A. M. Tyrrell, Sci. Rep. 2016, 6, 29581.

19 K. Lund, A. J. M anzo, N. Dabby, N. M ichelotti, A. Johnson-Buck, J. Nangreave, S. Taylor, R. Pei, M. N. Stojanovic, N. G. Walter, E. Winfree, H. Yan Nature 2010, 465, 206.

20 P. A. Rasheed, N. Sandhyarani, M icrochim. Acta 2017, 184, 981.

21 K. A. Willets, R. P. V. Duyne, Annu. Rev. Phys. Chem. 2007, 58, 267.

22 J. J. M ock, R. T. Hill, A. Degiron, S. Zauscher, A. Chilkoti, D. R. Smith, Nano Lett. 2008, 8, 2245.

23 F. Cannone, G. Chirico, A. R. Bizzarri, S. Cannistraro, J. Phys. Chem. B. 2006, 110, 16491. 
24 A. W. M. Haining, M. von Essen, S. J. Attwood, V. P. Hytönen, A. d. R. Hernández, ACS nano 2016, 10,6648.

25 F. Margadant, L. L. Chew, X. Hu, H. Yu, N. Bate, X. Zhang, M. Sheetz, PLoS Biol 2011, 9, e1001223.

26 R. J. Russell, P. S. Kerry, D. J. Stevens, D. A. Steinhauer, S. R. M artin, S. J. Gamblin, J. J. Skehel, PNAS 2008, 105, 17736.

27 S.-Y. Chen, J. J. Mock, R. T. Hill, A. Chilkoti, D. R. Smith, A. A. Lazarides, ACS nano 2010, 11, 6535.

28 M. W. Knight, Y. Wu, J. B. Lassiter, P. Nordlander, N. J. Halas, Nano Lett. 2009, 9, 2188.

29 C. Lumdee, S. Toroghi, P. G. Kik, ACS nano 2012, 6, 6301.

30 C. L. Du, Y. M. You, K. Johnson, H. L. Hu, X. J. Zhang, Z. X. Shen, Plasmonics 2010, 5, 105.

31 I. Vikhold-Lundin, S. Auer, M. Paakkunainen, J. A. E. M äättä, T. M unter, J. Leppiniemi, V. P. Hytönen, K. Tappura, Sens. Actuators B 2012, 171-172, 440.

32 J. J. Storhoff, R. Elghanian, R. C. Mucic, C. A. Mirkin, R. L. Letsinger, J. Am. Chem. Soc. 1998, 120, 1959.

33 C. A. M irkin, R. L. Letsinger, R. C. M ucic, J. J. Storhoff, Nature 1996, 382, 607.

$34 \mathrm{H}$. Liang, J.-P. Tuppurainen, J. Lehtinen, T. Viitala, M. Yliperttula, Eur. J. Pharm. Sci. 2013, 50, 492.

35 K. Niegelhell, S. Leimgruber, T. Grießer, C. Brandl, B. Chernev, R. Schennach, G. Trimmel, S. Spirk, Langmuir 2016, 32, 1550.

36 J. He, M. Lu, X. Zhou, J. R. Cao, K. L. Wang, L. S. Liao, Z. B. Deng, X. M. Ding, X. Y. Hou, S. T. Lee, Thin Solid Films 2000, 363, 240.

37 N. M. Green, N.M. Adv. Protein Chem. 1975, 295, 85.

38 N. M, Green, M ethods Enzymol. 1990, 184, 51.

39 Y. Uchiho, M. Shimojo, K. Furuya, K. Kajikawa, J. Phys. Chem. C 2010, 114, 4816.

40 T. Akimoto, S. Sasaki, K. Ikebukuro, I. Karube, Biosens. Biolectron. 2000, 15, 355.

41 X. Hong, E. A. H. Hall, Analyst 2012, 137, 4712.

42 A. Lundgren, B. Agnarsson, R. Zirbs, V. P. Zhdanov, E. Reimhult, F. Höök, ACS nano 2016, 10, 9974.

43 S. J. Hurst, A. K. Lytton-Jean, C. A. M irkin, Anal. Chem. 2006, 78, 8313.

44 K. K. Caswell, J. N. Wilson, U. H. F. Bunz, C. J. M urphy, J. Am. Chem. Soc. 2003, 125, 13914.

45 A. Dathe, M. Ziegler, U. Hübner, W. Fritzsche, O. Stranik, Nano lett. 2016, 16, 5728 .

46 C. Lumdee, B. Yun, P. G. Kik, ACS Photonics 2014, 1, 1224.

47 J. D. Driskell, R. J. Lipert, M. D. Porter, J. Phys. Chem. B. 2006, 110, 17444. 